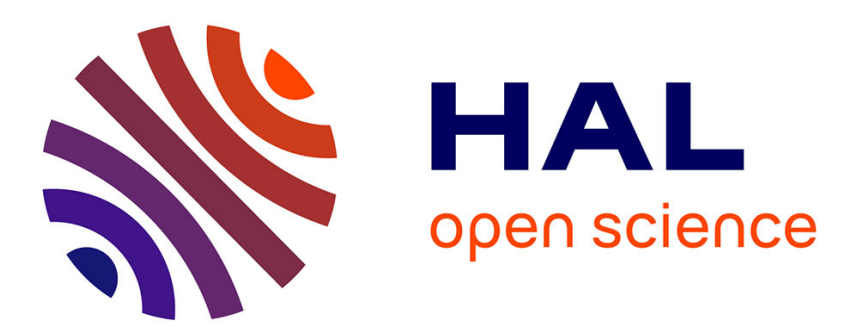

\title{
Social Representations of Digital Health Technology in different contexts: Why People Keep or Quit Using It?
}

Géraldine Escriva-Boulley, Camille Bernetière, Marie Préau, Françoise Paquienséguy, Tanguy Leroy

\section{To cite this version:}

Géraldine Escriva-Boulley, Camille Bernetière, Marie Préau, Françoise Paquienséguy, Tanguy Leroy. Social Representations of Digital Health Technology in different contexts: Why People Keep or Quit Using It?. 2021. hal-03163023

\section{HAL Id: hal-03163023 https://hal.science/hal-03163023}

Preprint submitted on 9 Mar 2021

HAL is a multi-disciplinary open access archive for the deposit and dissemination of scientific research documents, whether they are published or not. The documents may come from teaching and research institutions in France or abroad, or from public or private research centers.
L'archive ouverte pluridisciplinaire HAL, est destinée au dépôt et à la diffusion de documents scientifiques de niveau recherche, publiés ou non, émanant des établissements d'enseignement et de recherche français ou étrangers, des laboratoires publics ou privés. 
Social Representations of Digital Health Technology in different contexts: Why People Keep or Quit Using It?

Running title: Digital Health Technology and Social Representations in cancer context

Authors:

Géraldine Escriva-Boulley ${ }^{1}$

Camille Bernetière ${ }^{2}$

Marie Préau ${ }^{1}$

Françoise Paquienseguy ${ }^{2}$

Tanguy Leroy ${ }^{1}$

${ }^{1}$ Laboratory: Social Psychology Research Group [Groupe de Recherche en Psychologie Sociale] (EA 4163)

Psychology Institute [Institut de Psychologie] -University of Lyon

${ }^{2}$ Laboratory: Information and Communication Sciences Lyon Research Team [Équipe de Recherche de Lyon en Sciences de l'Information et de la Communication] (EA 4147) Institute of Human Sciences [Institut des Sciences de 1'Homme] - University of Lyon

Corresponding author

Géraldine Escriva Boulley

Groupe de Recherche en Psychologie Sociale (EA 4163)

Institut de Psychologie - Université Lyon 2

Bâtiment V (1er étage)

5 avenue $P$. Mendès-France

69676 - Bron (France)

escrivageraldine@gmail.com 


\section{$1 \quad$ Abstract}

Objectives: The use of digital health technologies (DHT) is increasingly

3 ubiquitous in intervention studies aimed at reducing health risks or improving the

4 management of chronic diseases such as cancer. However, although DHT clearly show

5 promises for a variety of applications, one third of users quit using DHT less than six

6 months after the purchase, which may limit their effectiveness. This study aims to identify

7 social representations (SR) of DHT, and to highlight why individuals adopt or are

8 reluctant to adopt DHT, as well as the reasons for their drop-out.

Methods: Five focus groups were lead with 18 participants (Mage $=43.72$ years,

10 Women $=13$ ) whose personal uses of DHT were heterogeneous and controlled. They

11 completed three tasks designed to elicit a wide range of SR of DHT.

Results: Results showed that individuals' concerns about DHT were focused on

13 four themes: 1) health versus well-being purposes, 2) price, 3) data protection and, 4)

14 difficulties for the elderly. The main reason for adopting DHT was that their use met a

15 need, an interest. Most participants made a fairly strong distinction between the DHT

16 which promote health and those promoting wellbeing. Reasons for reluctance and drop-

17 out were related to a lack of knowledge, information, transparency and mastery.

18 Conclusions: These findings may help DHT designers to understand what kind of

19 information are needed and relevant to users. This study also highlights users' SR of

20 DHT, as well as their expectations and fears which should be taken into account when

21 implementing interventions.

Keywords: cancer, drop-out, focus group, health, internet of things, maintenance, 


\section{Background}

The use of digital technologies (i.e., computer programs, websites, smartphone

3 applications or wearable devices) is increasingly ubiquitous in health intervention studies

4 (1). The increase of users, its economic feasibility and round-the-clock availability makes

5 it a potentially viable option in a wide variety of health settings (2) from risk reduction

$6(3,4)$ to the management of chronic diseases $(5)$. In the context of cancer, studies about

7 digital health technologies (DHT) is particularly relevant because the potential for DHT

8 to help patients manage cancer is accepted (6), few tailor-made cancer-focused DHT

9 currently exist, but several DHT for cancer are under development. Thus there is a need

10 to investigate this field of research. Otherwise, DHT may thus be used by individuals who

11 suffer from a disease as well as those who do not but aim to avoid illness or seek

12 wellbeing. For instance, studies highlighted the efficacy of DHT to manage sides effects

13 of cancer treatment (7) or to improve physical activity (3).

14 Although, DHT have the potential to personalize healthcare, to allow autonomous

15 health management, to change health behaviors and to promote well-being, one third of

16 users quit using DHT less than six months after the purchase (e.g., 7-10), which may

17 limit their effectiveness. Therefore, there is a need to better understand why individuals

18 adopt or are reluctant to adopt DHT, as well as the reasons for their drop-out. To enhance

19 the knowledge about these reasons is important to design better health interventions and

20 increase their effectiveness.

21 Most previous studies which investigated these reasons did it from the perspective

22 of healthcare providers. In contrast, studies conducted with users are scarce (12). Classic

23 behavior theories (e.g., technology acceptance model, the theory of planned behavior)

24 were used to identify the reasons that may lead to the use or the intention to use DHT. 
1 For instance, using a survey, a unified theoretical model identified five key factors:

2 performance expectancy, effort expectancy, social influence, facilitating conditions, and

3 threat appraisals (12). These factors are also highlighted and completed by data from non-

4 theoretically based studies about the use of DT in general, or DHT especially which used

5 surveys $(8,13,14)$, interviews (10) and comments posted on online marketplaces (15).

6 According to these studies, users start and keep using DHT because of perceived benefits,

7 potential DHT features enhancement $(10,15)$, curiosity and novelty $(10)$ and because they

8 had developed a routine of using it $(8,10)$. On the contrary, attrition was due to their

9 passive nature, appearance and obtrusiveness, the high frequency maintenance $(10,13)$,

10 the lack of users' needs (e.g., users feel that they do not really need it $(10,15))$, the lack

11 of knowledge about DHT and how to access and use the data provided (10), and concerns

12 about data sharing (14). To the best of our knowledge no study investigated reasons for

13 reluctance towards adopting DHT.

14 These studies have examined behaviors related to DHT regardless to individuals'

15 characteristics. However, research showed that users' gender as well as health related

16 factors and experiences may influence the use of DHT (16). In the same vein, none of

17 these studies investigated social representations (SR) related to HDT. SR are sets of ideas

18 or opinions consensually shared by a social group about a same social object $(16,17)$. By

19 studying SR, one may gain access to significant practices which may only be understood

20 in their specific cultural and social contexts granting them relevance and legitimacy (17).

21 To investigate SR, the focus group (FG) methodology have proven to be relevant

22 (18) as it provides a less interaction with the researcher, it enables peer-to-peer discussion

23 and it allows participants to freely react to each other's responses, thus permitting a deeper

24 insight into individuals' behaviors and experiences. In addition, FG methodology requires 
1 grouping participants according to targeted characteristics, allowing researchers to

2 observe the confrontation of several socio-cultural contexts. To the best of our

3 knowledge, no study has used such a methodology so far to examine SR of DHT. Using

4 this qualitative individual-centered design, the aim of this study is to examine SR related

5 to adoption, reluctance to adopt, and attrition of the use of DHT, considering health status,

6 experiences with DHT and health habits. Such a study design has been chosen to ensure

7 a strong diversity of the collected opinions rather than to test specific hypotheses. Our

8 only hypothesis is that some SR are common and should be found in every FG, showing

9 their stability and their potential reproducibility.

11 Methods

12 Participants

13 Participants were enrolled via an online collaborative research internet platform

14 dedicated to cancer. All participants were provided with full ethical information about the

15 study, signed a consent form and were invited to complete an anonymous screening 16 survey.

17 Based on the latter, a purposive sampling was performed (gather individuals with 18 shared characteristics). Such a sampling provides rich data relevant to the research 19 question $(18,19)$ as it guarantees the heterogeneity of opinions expressed. Using this 20 strategy, five FG were designed, including five to seven participants (supplementary 21 material Table 1 for details). The "health status" group (FG1) included participants taken into account cancer conditions, the "use of DHT for men" (FG2) and "use of DHT for women" (FG3) included participants who owned DHT taken into account their frequency

24 of use and the gender, the "quantified-self" group (FG4) took into account practice of 
1 quantified-self and the "physical activity" group (FG5) took into account the level of

2 physical activity. Physical activity was chosen as a target health behavior because of its

3 impact on health, and because it is the most targeted health behavior in DHT settings (20).

$5 \quad$ Protocol used during focus group

6 First, the purpose and the proceeding of the FG were reminded. Discussions were

7 moderated by a trained researcher. Participants were invited to perform three activities.

8 They were asked what DHT mean for them, and then they chose images of DHT and

9 explained their choice (supplementary material Table 2 for details). The "personas"

10 method was also used (20). Personas are archetypical characters (potential users)

11 presented with information about their needs, behaviors, and preferences (21). Although

12 persona are fictional, they represent generalizations of key characteristics and goals of

13 potential users (22) (supplementary material Figure 1 for example). All FG were

14 transcribed verbatims from videotapes of the sessions and anonymized.

\section{Data availability statement}

16 Data are available from the corresponding author upon reasonable request.

\section{Data analysis}

A thematic analysis (23) was performed to explore participants' SR of DHT, as

19 well as their reasons to adopt, avoid, or drop-out DHT according to their personal

20 experience. Transcripts were closely examined and descriptive codes were used to

21 identify key emergent themes and ideas related to the study objectives. Codes were then

22 gathered into higher-order concepts or subdivided as understanding progressed.

23 Descriptive accounts were produced to explore the content of key codes and comparing

24 data across groups and individuals. A double coding as well as an iterative review of 
1 coding and themes were performed to ensure the reliability, accuracy, and consistency of

2 the analysis.

\section{Results}

Of the 72 screened participants, 25 finally agreed to participate to the scheduled

5 FGs and 18 of them, aged 26 to 65 years, actually participants to FG ( $M_{\text {age }}=43.72$ years,

$6 S D_{\text {age }}=12.75$ years, $N_{\text {women }}=13$ ). Twelve participants owned a DHT (health and sport),

712 practiced physical activity and 13 had no personal history of cancer (Table 1). Except

8 for $\operatorname{sex}\left(\chi^{2}=5.12 ; p=.02\right)$, individuals who participate to FG did not differ from other 54

9 participants.

The common themes emerging across groups and accounting for the adoption, the reluctance to adopt, and the attrition of the use of DHT have been grouped into four key themes: 1) health versus well-being purposes, 2) price, 3) data protection, and 4)

14 difficulties for the elderly.

Health versus well-being purposes

This first theme deals with a distinction participants drew between DHT specifically targeting health and those addressing well-being: "Compare to DHT for wellbeing, DHT for health are serious. We are not looking for fun, here (FG5)". This SR

20 of DHT is used by participants to explain the adoption and the drop-out of DHT according

21 to its necessity versus futility. DHT for health are for individuals with a health condition or a disease, not for healthy ones: "If you are healthy there is no interest, but if you have

23 a disease and you need to check things every day it may be practical (FG5)". On the

24 contrary, ill patients are supposed to need DHT as they are beneficial: "He is sick, thus 
1 his relation [to DHT] changed, he has an everyday obligation (FG2)”; "[DHT] helps

2 individuals in their disease or monitor their disease. (FG4)". On the contrary, DHT for

3 wellbeing are viewed as less necessary: "There is a futile aspect. [...] it may be a cause

4 of drop-out. For me [DHT] are more for wellbeing are gadgets (FG4)". When talking

5 about DHT for wellbeing, participants highlighted the fun and social aspects as much as

6 the frequency of use: "One can use DHT punctually, [...], not for an everyday use

7 (FG1)”. However, participants acknowledged the role of DHT promoting wellbeing as

8 an opportunity for preventing diseases: "In my mind this is for individuals who have a

9 disease. But at the same time, it could permit to prevent disease, for physical monitoring

10 (FG2)". Besides, it is noteworthy that participants of the "health status" group (FG1)

11 made a less clear distinction between DHT dedicated to health or wellbeing than those of

12 other FGs. Indeed, they viewed DHT for health and wellbeing as being as much useful

13 but having different purposes "After cancer, [DHT] should be used more to improve

14 lifestyle and less for medical follow-up (FG1)”. FG1 is also the only group who discussed

15 the opportunity of using DHT to communicate with other health professionals than

16 doctors.

Price

All participants evoke price as a reason for their reluctance to use DHT: "I wait 20 for the price to decrease to buy it (FG1)". Several SR are associated to this issue. First, 21 participants mentioned the quality/price ratio: "The more expensive, the more reliable it is (FG5)", "It's expensive and it doesn't work as well as expected (FG3)”. Second, they

23 pointed out the usefulness/price ratio: "I'm not convinced of the utility and [...] the 24 relationship between utility and what it will cost (FG3)". Due to this price issue, 
1 according to participants, buying DHT is not possible for everyone: "The price is too

2 high, at 20 years old you can't buy a DHT at 400-800 euros (FG3)'”. Finally, participants

3 proposed a solution to overcome this barrier: "The question of price is a very interesting 4 one to rise. It's reserved for those who can afford it, there's no reimbursement (FG4)",

5 "Health insurance authorities should reimburse more for sick people (FG2)".

6

7 Data protection

This theme led to the same controversies and nuanced opinions in each FG. All

9 participants raised the issue of data protection, especially when it came to health data.

10 Their concerns were about what was done with these data, who uses them and to what

11 purpose: "DHT collect data and stock it somewhere, it's frightening (FG5)", "This is not only altruist [...] or for one's health [...] I think there is something else, but I can't define it (FG1)”. Some respondents feared that their health data may be turned against themselves: "This is awesome for health insurance authorities. [About the connected toothbrush] they can see he didn't brush his teeth, they will not reimburse his dental care (FG2)", "Imagine... you have hidden your disease to your boss, and he finds out (FG5)". However, data collection was not systematically perceived as a threat: "I don't care [about data protection] (FG3)”. Indeed, participants view ways to protect their data: “There is a lot of solutions to protect your data (FG4)" and consider DHT as possibly 20 helpful for health research: "In health, it doesn't bother me [...]. I believe that [...], it has a long-term use, it can help other people (FG2)”. However, this agreement for sharing data only concerns health data, not wellbeing data: "[...] in a disease setting it's ok, but when you jog every day, it's personal (FG5)". 


\section{Difficulties for the elderly}

Participants commonly assumed that the elderly experienced more difficulties in using DHT than the youths. One reason is that DHT evolve faster than the elderly can follow "It's a little disturbing at first, when you are not used to it (FG2)", "She thinks it's going too fast, and she's too old (FG3)". That explains reluctance -to smartphone technology- even when other DHT are used: "For instance, my father uses a laptop, but he doesn't want a smartphone (FG1)". However, participants highlighted that DHT may be useful for the elderly: "It could be useful, when you go on a walk for instance, [...] when you have a certain age you have to be careful about your [physical activity] leisure (FG5)". To this purpose, all participants agreed that the elderly need training: "They are afraid of [technological] progress because they are not enough accompanied, there is a lack of pedagogy (FG4)”.

\section{Discussion and Conclusions}

DHT are presented as a real opportunity to improve health and wellbeing as they may lead individuals to reflect on themselves, based on data from their behaviors, and as a consequence, DHT may help people change their health behaviors. However, the use of DHT does not last long, which may limit their effectiveness on health. Using a focus groups methodology, the aim of this study was to highlight some SR of DHT, and to identify what individuals focus their attention on as well as their beliefs and practices in regards to DHT.

Results have shown that four themes were particularly important for participants: 1) health versus well-being purposes, 2) price, 3) data protection, and 4) difficulties for the elderly. Individuals thus seem to focus their attention on these themes to assess DHT 
1 and decide to adopt them, maintain their use or drop them out. Actually, when it comes

2 to assess the usefulness of a DHT, there is no validated comprehensive tool to help

3 individuals to identify which ones are effective and of high quality (24). These four

4 themes may be added to the assessment criteria proposed by McKay et al. (24), namely a

5 description of the usability and functionality, a critique of the potential to promote

6 behavior change, and the quality of the health-related content. In other words, potential

7 users should be able to access information about whether the DHT is designed to improve

8 health or wellbeing, what are its usability, functionality and effectiveness considering its

9 price, and how data protection is ensured. Moreover, people should easily get access to

10 an educational and accessible tool about "how to" fully use the DHT (e.g., tutorial, short

11 notice of use). Besides, through the four themes found in this study, the reasons for the

12 adoption, reluctance, and drop-out of DHT may be highlighted.

13 Reasons to adopt a DHT

14 To adopt a DHT, its use must be meaningful for individuals. For instance, in the

15 present study adopting and keeping using a DHT makes more sense when it is designed

16 to improve health rather than wellbeing. This result is consistent with previous results

17 showing that individuals with a health condition are more prone to use health related

18 resources alike DHT (25). In addition, participants emphasized that in case of disease, the

19 use of DHT may prove to be of vital necessity. Another interesting result of the present

20 study is that, while most participants make a fairly strong distinction between DHT

21 according to their purpose (health versus wellbeing), participants from our health status

22 group agreed to consider health and well-being as the two sides of a same coin. Indeed,

23 for individuals who suffer or have suffered from a disease such as cancer, wellbeing is

24 clearly one of the issues of the care protocol. 
Although some kind of interest is needed to adopt a DHT designed to improve

2 wellbeing, participants also found that using such a DHT was more joyful than using a

3 DHT designed to improve health, which is viewed as more serious. Furthermore they saw

4 DHT designed to improve wellbeing to lead to social relation with family and friends,

5 while DHT designed to improve health lead to relation with health professionals.

6 Participants also emphasized the difference between the need for an everyday use of a

7 DHT designed to improve health and the punctual use of a DHT improving wellbeing.

8 These results are in accordance with the data from a previous study highlighting that

9 behaviors related to the use of DHT should be seen as a dynamic process going beyond

10 the simple distinction between adoption and drop-out (26). The irregular practice, named

11 intermittent discontinuance, has to be taken into account as the efficacy of a DHT depends

12 on its continued usage to keep track of users' health status and efficiently provide health

13 advice (26). Determinants of intermittent discontinuance are neutral satisfaction (i.e., the

14 use of the DHT produces neither pleasure or displeasure), neutral disconfirmation (i.e.,

15 achieving the minimum instead of the desired expectation from the DHT) and attitudinal

16 ambivalence (i.e., difficulty for users to clearly decide if assessments of a DHT are

17 positive or negative) (26). Although, the present study increases knowledge about these

18 variables by providing a better understanding of users' representations and expectations

19 of DHT, there is a need to examine further, whether the purpose of the DHT (improve

20 health or wellbeing), data protection, price and age of the users affect behaviors related

21 to use of DHT.

Reasons to be reluctant and to drop-out DHT

Reluctance to adopt DHT and drop-out were related to a lack of knowledge,

24 information, transparency and mastery of the DHT. For instance, they need to know how 
1 to use DHT, what use they can have of them, how to interpret data and what happen to

2 data. Compared to DHT designed to improve health, in which patients are quite well

3 accompanied in their use of the technology, users of DHT focused on wellbeing, have to

4 figure out by themselves how it works and why it is important. Although several studies

5 have shown that the elderly refrain from using DHT because of their lack of knowledge

6 (e.g., 30,31), all users also face a readability issue (24): information from DHT may be

7 misunderstood, viewed as harder to understand than those obtained with unconnected

8 objects, which may lead people to drop-out DHT. Besides, DHT designed to improve

9 wellbeing are considered to be fun, easy to understand, and easy to change for another

10 DHT. Therefore, information provided by such tools are not viewed as relevant to

11 improve health, broadly speaking. In a previous study, that point was described as a

12 reason to drop-out the DHT (29) whereas in the present study, it is also a reason for

13 reluctance to adopt a DHT. Hence, there is a need to educate people about the

14 opportunities that DHT could offer to improve health and wellbeing, to highlight the

15 necessity of a continuous use of DHT, and to help users to better understand the data

16 generated. Future studies should fulfill each of these requirements to enhance

17 maintenance and effectiveness of DHT.

18 The data protection issue has already been underlined in several studies (e.g.,

19 13,25,27). Consistently, present results highlight participants' concerns about what is

20 done with their data, who uses them, and to what purpose. These are reasons both for

21 reluctance to adopt DHT and drop-out. However, the present study brings an innovative

22 result: all participants did not share such fears. Some participants did not perceive the

23 threat and were quite confident about DHT security. Furthermore, individuals were more

24 prone to widely share health data, considering it may be helpful for health research. 
1 Complementary and quantitative studies are needed to examine whether these reasons

2 may affect the maintenance, reluctance or the drop-out of DHT.

3 Study limitations

While the present study brings new valuable knowledge to the field, this study

5 also has several limitations. First, the sample size is quite small while acceptable for a

6 qualitative study. However, data are consistent with results from previous studies and the

7 focus groups methodology has been helpful to highlight innovative results. Future studies

8 should acknowledge these findings and assess their actual relevance. Second, participants

9 were recruited from an internet platform that originally targeted breast cancer patients,

10 cancer survivors and their families. Thus, these three profiles were present in our sample,

11 however participants were mostly female and healthy. Despite our efforts to constitute

12 the most heterogeneous focus groups possible, the SR observed may not reflect those of

13 all individuals. As a consequence, further research is needed to assess the

14 representativeness of the SR reported in the present study.

15 Practical Implications

16 DHT aiming to improve health may be a part of a care protocol, helping patients

17 manage a high volume of information and self-management tasks, facilitating the

18 coordination and synthesis of information from different providers and about different

19 conditions and the patients-care providers' communication, and support patients in their

20 roles of self-advocate and expert (30). These possibilities have been reported by

21 participants as easing the adoption and maintenance of DHT (30). Otherwise, patients

22 who suffer from cancer can benefit from DHT for health as well as for wellbeing. For

23 instance, in the present study, participants used a pedometer apps or relaxation apps

24 during or between treatments. 


\section{General Conclusion}

2

This study provides an in-depth understanding of the SR of DHT. Individuals'

3 concerns leading them to adopt or not a DHT and to keep using it or not are: its benefits

4 for health, its price, how it ensures data protection, and its usability. These findings may

5 help DHT designers to understand what kind of information is needed and relevant to

6 potential users. This study also highlights users' expectations and fears that should be

7 taken into account when implementing interventions in health care setting involving

8 DHT.

9 
1 Acknowledgment: This work was supported by the Metropole de Lyon grant. The sample

2 was recruited via an internet platform named Seintinelles (Equivalent of USA-based

3 “Army of Women" platform).

$4 \quad$ National ethical committee approval: $\mathrm{n}^{\circ} \mathrm{RCB} 2017-\mathrm{A} 03360-53$

5 Conflict of interest: Authors state no conflict of interest.

6 


\section{References}

1. Michie S, Yardley L, West R, Patrick K, Greaves F. Developing and evaluating digital interventions to promote behavior change in health and health care: Recommendations resulting from an international workshop. J med internet res, 2017; 19(6): e232. doi: 10.2196/jmir.7126

2. Rehman H, Kamal AK, Sayani S, Morris PB, Merchant AT, Virani SS. Using mobile health (mHealth) technology in the management of diabetes mellitus, physical inactivity, and smoking. Cur atheros rep, 2017; 19: 16. doi: $10.1007 / \mathrm{s} 11883-017-0650-5$

3. Schoeppe S, Alley S, Rebar AL, Hayman M, Bray NA, Van Lippevelde W, et al. Apps to improve diet, physical activity and sedentary behaviour in children and adolescents: a review of quality, features and behaviour change techniques. Int $\mathbf{J}$ of Behl Nut Phy Act, 2017; 14 (1): 83. doi: 10.1186/s12966-017-0538-3

4. Payne HE, Lister C, West JH, Bernhardt JM. Behavioral Functionality of Mobile Apps in Health Interventions: A Systematic Review of the Literature. JMIR mHealth and uHealth, 2015;3(1):e20. doi: 10.2196/mhealth.3335

5. Jones KR, Lekhak N, Kaewluang N. Using mobile phones and short message service to deliver self-management interventions for chronic conditions: A metareview. Worldvs on Evid-Based Nurs. 2014; 11(2): 81-88. doi 10.1111/wvn.12030

6. Odeh B, Kayyali R, Nabhani-Gebara S, Philip N. Optimizing cancer care through mobile health. Supe Care Cancer. 2015; 23: 2183-2188. doi: 10.1007/s00520-015$2627-7$

7. Escriva Boulley G, Leroy T, Bernetière C, Paquienseguy F, Desfriches-Doria O, Préau M. Digital health interventions to help living with cancer: A systematic 
review of participants' engagement and psychosocial effects. Psycho-Oncology. 2018; 27(12): 2677-2686. doi: 10.1002/pon.4867

8. Ledger D, McCaffrey D. Inside Wearables, How the Science of Human Behaviour Change Offers the Secret to Long-Term Engagement. [Internet], 2014 [cited 2019 april 29].Available from: https://medium.com/@endeavourprtnrs/inside-wearablehow-the-science-of-human-behavior-change-offers-the-secret-to-long-termengagement-a15b3c7d4cf3

9. Tate DF, Zabinski MF. Computer and Internet Applications for Psychological Treatment: Update for Clinicians. J of Clin Psych 2004; 60: 209-220. doi: 10.1002/jclp.10247

10. Lazar A, Koehler C, Tanenbaum J, Nguyen DH. Why we use and abandon smart devices. In: Proceedings of the 2015 ACM International Joint Conference on Pervasive and Ubiquitous Computing - UbiComp '15. 2015: 635-646. doi:10.1145/2750858.2804288

11. Wright SP, Hall Brown TS, Collier SR, Sandberg K. How consumer physical activity monitors could transform human physiology research. Am J Physiol Regul Integr Comp Physiol. 2017; 312(3): 358-367. doi:10.1152/ajpregu.00349.2016

12. Sun Y, Wang N, Guo X, Peng Z. Understanding the acceptance of mobile health services: a comparison and integration of alternative models. J of Elec Com Res 2013; $\quad$ 14(2): $\quad$ 183-200. $\quad$ Available https://search.proquest.com/docview/1372758336?accountid=11077

13. Shih PC, Han K, Poole ES, Rosson MB, Carroll JM. Use and Adoption Challenges of Wearable Activity Trackers. In iConference Proc. 2015;(1):1-12. Available 
from

https://www.ideals.illinois.edu/bitstream/handle/2142/73649/164_ready.pdf

14. Epstein DA, Caraway M, Johnston C, Ping A, Fogarty J, Munson SA. Beyond Abandonment to Next Steps: Understanding and Designing for Life after Personal Informatics Tool Use. Proc SIGCHI Conf Hum Factor Comput Syst. 2016; 165(7): 1109-1114. doi: $10.1145 / 2858036.2858045$

15. Clawson J, Pater JA, Miller AD, Mynatt ED, Mamykina L. No longer wearing: investigating the abandonment of personal health-tracking technologies on craigslist. In: Proceedings of the 2015 ACM International Joint Conference on Pervasive and Ubiquitous Computing - UbiComp '15. 2015: 647-658. doi: $10.1145 / 2750858.2807554$

16. Hermsen S, Moons J, Kerkhof P, Wiekens C, De Groot M. Determinants for Sustained Use of an Activity Tracker: Observational Study. JMIR mHealth uHealth. 2017; 5(10): e164. doi: 10.2196/mhealth.7311

17. Caillaud S, Kalampalikis N. Focus Groups and Ecological Practices: A Psychosocial Approach. Qual Res Psychol. 2013;10(4): 382-401. doi: $10.1080 / 14780887.2012 .674176$

18. Côté L, Turgeon J. Appraising qualitative research articles in medicine and medical education. Med Teach. 2005; 27(1): 71-75. doi: $10.1080 / 01421590400016308$

19. Giacomini MK, Cook DJ. Users' guides to the medical literature: XXIII. Qualitative research in health care A. Are the results of the study valid?. JAMA J Am Med Assoc. 2000; 284(3): 357-362. doi:10.1001/jama.284.3.357

20. Cooper A. The Inmates Are Running the Asylum: Why High Tech Products Drive 
Us Crazy and How to Restore the Sanity. Sams; 1999.

21. Chapman CN, Milham RP. The Personas' New Clothes: Methodological and Practical Arguments against a Popular Method. In Proceedings of the human factors and ergonomics society annual meeting. Sage CA: Los Angeles, CA : SAGE Publications; 2006: 634-636.

22. Madsen A, Mckagan SB, Sayre EC, Martinuk MA, Bell A. Personas as a Powerful Methodology to Design Targeted Professional Development Resources Methodology : Creation of Personas. 2014; preprint arXiv:1408.1125.

23. Braun V, Clarke V. Using thematic analysis in psychology. Qual Res Psychol. 2006; 3(2): 77-101.

24. McKay FH, Cheng C, Wright A, Shill J, Stephens H, Uccellini M. Evaluating mobile phone applications for health behaviour change: A systematic review. J of telemed telecare. 2018; 24(1): 22-30. doi: 10.1177/1357633X16673538

25. Fox S, Duggan M. Pew Internet \& American Life Project. The Diagnosis Difference. 2013 [cited 2019 april 29]. Available from:http://www.pewinternet.org/Reports/2013/The-DiagnosisDifference/Summary-of-Findings.aspx.

26. Shen XL, Li YJ, Sun Y. Wearable health information systems intermittent discontinuance: A revised expectation-disconfirmation model. Ind Manag Data Syst. 2018; 118(3): 506-523. doi 10.1108/IMDS-05-2017-0222

27. Czaja SJ, Charness N, Fisk AD, Hertzog C, Nair SN, Rogers WA, et al. Factors predicting the use of technology: Findings from the Center for Research and Education on Aging and Technology Enhancement (CREATE). Psychol Aging. 2006; 21(2): 333-352 
28. Zulman DM, Kirch M, Zheng K, An LC. Trust in the internet as a health resource among older adults: Analysis of data from a nationally representative survey. J Med Internet Res. 2011; 13(1): e19. doi: 10.2196/jmir.1552

29. Phillips B, Zhao H. Predictors of Assistive Technology Abandonment. Assist Technol. 1993; 5(1): 36-45. doi:10.1080/10400435.1993.10132205

30. Zulman DM, Jenchura EC, Cohen DM, Lewis ET, Houston TK, Asch SM. How Can eHealth Technology Address Challenges Related to Multimorbidity? Perspectives from Patients with Multiple Chronic Conditions. J Gen Intern Med. 2015; 30(8): 1063-1070 
Table 1. Characteristics of focus group participants

\begin{tabular}{lc}
\hline & $\mathrm{N}(\%)$ \\
\hline Number of participants & 18 \\
Mean age & 43.72 \\
Women & $13(72)$ \\
Illness & $7(39)$ \\
$\quad$ Have a chronic disease (other & \\
than cancer) & $1(5)$ \\
Have cancer & $4(22)$ \\
Had cancer & $13(72)$ \\
Never have cancer & \\
Own a digital technology & $15(83)$ \\
Have & $3(17)$ \\
Never have & $12(67)$ \\
Have a digital technology for & \\
health & \\
Frequency of use & $10(56)$ \\
Frequently & $4(22)$ \\
Regularly & $1(5)$ \\
Occasionally & $12(67)$ \\
Physical activity (yes) & \\
Frequency of physical activity & $5(28)$ \\
Frequently & $5(28)$ \\
Regularly & $2(11)$ \\
Occasionally & \\
\hline
\end{tabular}

